\title{
Spectrum-aware and Energy-adaptive Reliable Transport for Internet of Sensing Things
}

\author{
A. Ozan Bicen, Member, IEEE, Ozgur Ergul, Member, IEEE, Ozgur B. Akan, Fellow, IEEE
}

\begin{abstract}
Wireless sensors equipped with cognitive radio, i.e., cognitive radio sensor networks (CRSN), can access the spectrum in an opportunistic manner and co-exist with licensed users to mitigate the crowded spectrum problem and provide ubiquitous remote event monitoring and tracking for cyber-physical systems. In this paper, a novel transport layer protocol for CRSN, spectrum-aware energy-adaptive reliable transport (SERT) protocol is presented to enable energy-adaptive collaborative event sensing in spectrum-scarce cyber-physical systems. To the best of our knowledge, this is the first attempt to specifically devise a reliable event transport scheme for CRSN.
\end{abstract}

Index Terms-Opportunistic spectrum access, cognitive radio sensor networks, transport layer, distributed sensing.

\section{INTRODUCTION}

$\mathbf{C}$ OGNITIVE radio (CR) is the key enabling technology to provide opportunistic spectrum access (OSA) to increase spectrum efficiency by determining spectrum holes and communicating throught them [1]. When used in wireless sensor networks (WSN), OSA capability enables communication over licensed spectrum, which constitutes a new paradigm, i.e., cognitive radio sensor networks (CRSN) [2]. Overall, CRSN provides significant advantages over WSN, including utilization of multiple channels and spectrum adaptability. CRSN can be used to provide distributed sensing for cyber-physical systems, such as agriculture [3], disaster relief [4], smart grid applications [5]. However, realization of these applications depends on reliable and energy-efficient event transport.

In a typical CRSN architecture nodes can be in the communication range of different licensed users, i.e.primary users (PU). Therefore, they are expected to provide OSA capability while meeting a certain event detection reliability requirement. Union of WSN and CR pose various challenges for the realization of reliable event transport as intermittent communication,

Copyright (c) 2015 IEEE. Personal use of this material is permitted. However, permission to use this material for any other purposes must be obtained from the IEEE by sending a request to pubs-permissions@ieee.org.

This work was supported in part by the Turkish Scientific and Technical Research Council under grant \#110E249 and by the Turkish National Academy of Sciences Distinguished Young Scientist Award Program (TUBA-GEBIP).

A. O. Bicen is with the School of Electrical and Computer Engineering, Georgia Institute of Technology, Atlanta, GA 30332. (e-mail: bozan@ece.gatech.edu)

Ozgur Ergul is with the Department of Electronic and Communication Engineering, Cankaya University, Ankara 06790, Turkey. (e-mail: oergul@cankaya.edu.tr)

O. B. Akan is with Electrical Engineering Division, Department of Engineering, University of Cambridge, UK (e-mail: oba21@cam.ac.uk), and with the Next-generation and Wireless Communications Laboratory (NWCL), Department of Electrical and Electronics Engineering, Koc University, Istanbul,34450, Turkey (e-mail: akan@ku.edu.tr) spectrum mobility and network lifetime. Intermittent communication occurs because sensors must periodically pause event reporting while spectrum is sensed. During spectrum mobility, transport layer must be able to adapt to new parameters such as PU statistics and channel conditions quickly. Network lifetime is also crucial, since sensor nodes are resource-constrained. Both the number of reporting sensors and individual reporting rate of each sensor should be carefully designed to increase network lifetime while providing required event detection reliability. These challenges under processing limitations, dense deployment and bursty communication nature of sensor nodes call for a versatile and efficient transport layer to realize opportunistic distributed sensing for cyber-physical systems.

In this paper, spectrum-aware energy-adaptive reliable transport protocol, SERT is presented. SERT adapts to varying spectrum opportunities and energy constraints of CRSN. To the best of our knowledge, this is the first work on the transport layer focusing on reliable event transport for opportunistic distributed sensing. Distinctive features of SERT are:

1) Spectrum Awareness: SERT regulates sensor report rate by adapting to varying spectrum sensing duration caused by spectrum handoff.

2) Energy-adaptive Opportunistic Reliability: SERT chooses sensors and controls their rates to reach desired reliability in event observation under OSA by a proactive congestion avoidance mechanism.

3) Reliable Network Life Time: Sensor lifetime is increase by limiting energy consumption and intlligently choosing reporting sensors.

4) Low Complexity: SERT follows the decision interval based UDP-oriented protocols with enhancements tailored to address the unique requirements of the OSA, resulting in a low-complexity adaptive transport layer.

\section{RELATED WORK}

Recently, several challenges of CRSN have been tackled by the research community. Using partially observable Markov decision process, an energy-efficient channel assignment is studied in [6]. Furthermore, a two-stage spectrum sensing scheme to minimize spectrum sensing time is presented in [7], [8]. However, an event transport protocol that adapts itself to spectrum opportunities is yet to be developed for CRSN.

Problems related to transport layer have been investigated thoroughly for WSNs. In [9]-[11] proactive and predictive congestion avoidance approaches are proposed to prolong network lifetime. In [12], an end-to-end solution approach is proposed. In [13], [14], reliability in WSN is re-defined 


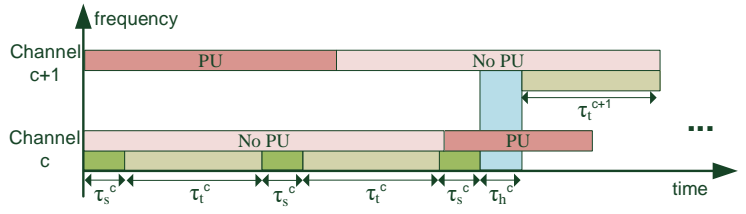

Fig. 1. Sensor node activity pattern with time.

based on event tracking accuracy objective at sink, instead of end-to-end packet-based reliability. Although these protocols address WSN requirements, they are shown to have very poor performance in CRSN [15].

By tuning TCP for CR compatibility, a set of transport protocols were derived from TCP in [16]. In [17] TCP CRAHN, which incorporates channel information and spectrum sensing function into the TCP rate control algorithm is proposed. Jointly addressing CR functionalities, modulation, coding, and frame size, cross-layer optimization schemes are proposed in [18], [19] to maximize TCP throughput. An equation-based transport protocol based on TCP Friendly Rate Control for Cognitive Radio (TFRC-CR) is proposed in [20]. It can adapt to the changes in spectrum by making use of the FCC mandated spectrum databases. A CR-aware transport protocol for MANETs that can handle drastic channel changes is proposed in [21]. However, authors do not investigate reliability issues.

In our prior work [15], we show that the existing transport protocols for WSN [9]-[14] exhibit very poor performance in CRSN. The dominant factor in this performance degradation is the spatio-temporally varying spectrum opportunities and imposed delay on communication due to spectrum sensing and handoff functionalities of cognitive radio [15].

There are only a small number of solutions targeting transport layer for the CRSN. An energy-efficient transport protocol for CRSN (RETP) is proposed in [23], that aims to prolong the lifetime of nodes. Due to the assumption that every node can reach the sink in one hop, RETP is not scalable. Opportunistic Hybrid Transport Protocol (OHTP) for Cognitive Radio Ad Hoc Sensor Networks is presented in [24]. It uses rate-based transmission under heavy congestion and window-based transmission otherwise. It also does not consider reliability in event signal delivery for CRSNs.

Although there is significant amount of work in transport protocols for WSN and rate control schemes for CRN, none of these address challenges regarding event transport in CRSN. Thus, a spectrum-aware transport protocol that meets distributed sensing requirements is needed for CRSN.

\section{CRSN NETWORK ARCHITECTURE}

\section{A. Spectrum Management Model}

Each node has a single transceiver with the capability to move between channels when a PU is detected. Sensors sense spectrum periodically. PU ON state means channel is occupied by PU. Channel occupation is modeled by a Poisson process. After event detection, samples taken from the event signal are collaboratively reported to the sink. Sink selects sensors that will participate in event reporting by estimation of distortion requirements and meeting energy constraints as explained in Section V and forms an estimate of the event after gathering sensor data. Sensor node behavior in a channel $c$ is modeled with data transmission $\left(\tau_{t}^{c}\right)$, spectrum sensing $\left(\tau_{s}^{c}\right)$ and spectrum handoff $\left(\tau_{h}^{c}\right)$ periods as shown in Fig. 1.

A detailed investigation of the effect of a wide range of parameters on the performance of CRNs is given in [25]. Effects of sensing time, PU activity and channel heterogeneity are investigated. We also investigate the effects of channel heterogeneity and incorporate the effects of spectrum sensing time when we calculate the reporting rate. In addition, we analyze its effects on reliability to maintain a certain distortion.

\section{B. Event-to-sensor Dispersion and Distributed Sensing Model}

A group of sensors, try to estimate the event signal collaboratively. Each observation $s_{m}(t)$ is the distorted version of event signal $\theta(t)$ by observation noise $\eta_{m}(t)$ and dispersion loss in signal power $\gamma_{m}(t)$ due to the distance between the sensor and the event source, at sensor $m$ and time $t$ i.e., $s_{m}(t)=\gamma_{m}(t) \cdot \theta(t)+\eta_{m}(t)$. For the estimation to cover a large number of event types, assumptions on noise must be established. Noise power may be measured by the nodes when there is no active event. Furthermore, environmental noise generally has zero mean. Except these first two moments, no more assumption must be made.

The sink collects samples during estimation interval $\left(\tau_{d}\right)$. Under adverse channel conditions, frequent spectrum handoffs, etc., the number of samples sent by the reporting nodes may not be sufficient to estimate the event signal with the desired accuracy. In such cases, nodes should send the rest of the data in the following data transmission interval. Therefore, estimation interval, $\tau_{d}$, may span a few data transmission, spectrum sensing and spectrum handoff durations.

Sufficient number of samples to meet a mean square error in event signal estimation is determined by Eqn (2). Since event signal SNR is different for each node, the number of samples varies for each node. Nodes with the greatest event signal SNR must be chosen, but battery level of nodes may dictate alternative node selections. Details are given in Section $\mathrm{V}-\mathrm{C}$ and Algorithm 1. The number of received samples during the $k^{\text {th }}$ estimation interval is found as

$$
X(k)=\tau_{d} \cdot \sum_{m=1}^{M} G_{m} \cdot S_{p}
$$

where $M$ is the number of the source nodes, $G_{m}$ is the packet reception rate from sensor $m, S_{p}$ is the number of

TABLE I

PARAMETER DEFINITIONS

\begin{tabular}{l|l||l|l}
$\tau_{t}$ & data transmission duration & $X(k)$ & rcvd smpls in $k^{t h}$ est. intrvl. \\
\hline$\tau_{s}$ & spectrum sensing duration & $D$ & distributed estimation distortion \\
\hline$\tau_{h}$ & spectrum handoff duration & $D_{0}$ & desired minimum distortion \\
\hline$\theta(t)$ & event signal & $X_{d}$ & desired samples to meet $D_{0}$ \\
\hline$\gamma_{m}(t)$ & dispersion loss & $r_{c}$ & channel utilization efficiency \\
\hline$\eta_{m}(t)$ & observation noisen & $\Gamma$ & reliability \\
\hline$s_{m}(t)$ & observed event signal & $\alpha$ & forgetting factor for EMWA \\
\hline$\tau_{d}$ & estimation interval & $M$ & \# active sensors
\end{tabular}




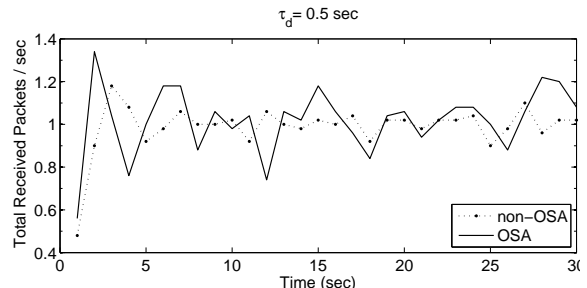

(a)

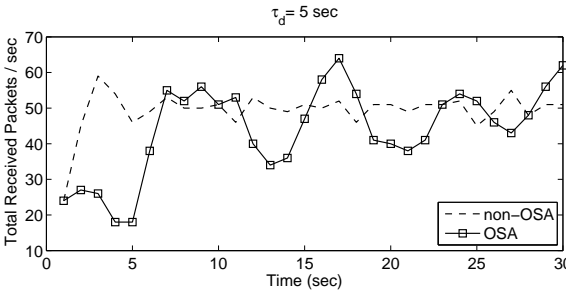

(b)

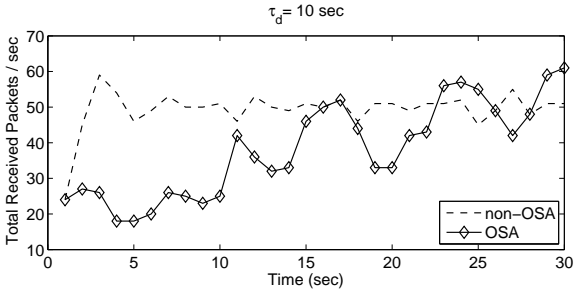

(c)

Fig. 2. Comparison of total number for received packets at sink for various $\tau_{d}$ values in OSA and non-OSA environments.

samples contained in a packet. The received sample vector at the end of $k^{\text {th }} \tau_{d}$ is a combination of sensing noise and sensed signal as $\mathbf{r}=\boldsymbol{\gamma} \cdot \boldsymbol{\theta}+\mathbf{n}$, where $\mathbf{r}=\left[r_{1}, \cdots, r_{X(k)}\right]^{\mathrm{T}}$, $\boldsymbol{\gamma}=\left[\gamma_{1}, \cdots, \gamma_{X(k)}\right]^{\mathrm{T}}, \boldsymbol{\theta}=\left[\theta_{1}, \cdots, \theta_{X(k)}\right]^{\mathrm{T}}$, and $\mathbf{n}=$ $\left[n_{1}, \cdots, \eta_{X(k)}\right]^{\mathrm{T}}$. Our linear, unbiased estimate will be of the form, $\hat{\theta}=\mathbf{a}^{T} \mathbf{r}$, such that, $E(\hat{\theta})=\theta$, where $\mathbf{a}$ is the weight vector we are trying to find, to use in the estimation and $E(\cdot)$ is expected value. For the estimate to be unbiased, $E(\mathbf{r})$ must be linear in $\theta$, i.e., $E(\mathbf{r})=\gamma \theta$. Replacing, we have $\mathbf{a}^{T} \boldsymbol{\gamma}=1$.

The best estimator under these conditions is the one with minimum variance which is the Best Linear Unbiased Estimator (BLUE) [26]. Mean square error (MSE) for BLUE is

$$
\begin{aligned}
D & =\operatorname{Var}[\hat{\theta}]=\left[\boldsymbol{\gamma}^{T} \mathbf{R}^{-1} \boldsymbol{\gamma}\right]^{-1} \\
& =\left(\sum_{n=1}^{X(k)} \frac{\gamma_{n}^{2}}{\xi_{n}^{2}}\right)^{-1}=\frac{\sigma_{\theta}^{2}}{\tau_{d} S_{p}}\left(\sum_{m=1}^{M} G_{m} \psi_{m}\right)^{-1}
\end{aligned}
$$

where $\psi_{m}$ is the signal-to-noise ratio $\left(\frac{\gamma_{m}^{2} \cdot \sigma_{\theta}^{2}}{\xi_{m}^{2}}\right)$ for samples of node $m$. We assume that during an estimation interval $\tau_{d}, \psi_{m}$ of a sensor does not change. SERT uses Eqn. (2) in formulation of energy-adaptive source node selection problem to calculate distributed estimation distortion $D$ for a set of selected source nodes and compare $D$ with desired distortion level $D_{0}$.

\section{FACTORS THAT EFFECT RELIABle EVEnt TRANSPORT}

In this section, we investigate the factors that have major impact on reliable event reporting. Once an available channel is found, the sink can have a solid estimate about how many samples per time can be expected from that node, i.e. date rate, and accordingly, it can adjust the number of required nodes to participate in event sensing. However, in OSA, PU may arrive any time. Therefore, all such estimations must be re-evaluated at the spectrum handoff. A transport protocol that aims reliable transfer of event data should handle the following factors:

- On PU arrivals, and changing channel conditions, a new channel must be used. Reporting rate of sensors must be adjusted to meet the minimum distortion. We explain how this is handled in SERT in the following section.

- There is a tradeoff in the choice of parameters such as spectrum sensing duration, tolerable distortion level, e.g., longer spectrum sensing duration results in more accurate sensing, but leaves less time for sensor data transmission,

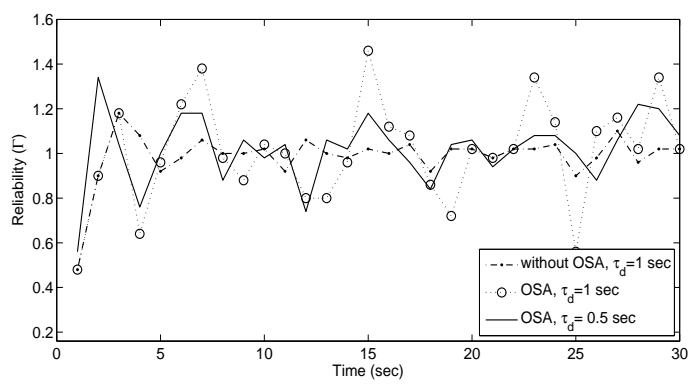

Fig. 3. Reliability performance at sink for $\tau_{d}=0.5 \mathrm{sec}$ with OSA and $\tau_{d}=1 \mathrm{sec}$ with OSA and without OSA.

which mandates more sensors to participate. We examine such effects in Section IV-B.

- Coordination is an important issue. Here, the tradeoff is, time allocated to coordination, e.g., to minimize the number of utilized nodes, vs. time for data transmission. We explain the effect of coordination in Section IV-C.

\section{A. Impact of OSA on Reliable Event Transport}

Fluctuations in reliability may be extreme due to the spectrum handoffs in CRSN. We first investigate the effect of intermittent communication on reliability via a case study. Variations on spectrum sensing duration causes fluctuation on reliability. Since, adaptations to reporting rate can only be done at the beginning of the next decision interval, long decision interval prolongs such adaptations. Therefore, performance changes by the varying $\tau_{d}^{c}$ as well $\tau_{t}^{c}$ and $\tau_{s}^{c}$.

We performed simulations by extending $n s-3$ [27]. A total of 100 sensor nodes and a sink are placed randomly in a $500 \mathrm{~m}$ $\mathrm{x} 500 \mathrm{~m}$ field. Source nodes are selected from an event radius of $30 \mathrm{~m}$, and transmit power $\left(P_{t}\right)$ is set to $10 \mathrm{dBm}$. Path loss exponent is taken as 3 , reference distance $1 \mathrm{~m}$, reference loss $55 \mathrm{~dB}$, noise floor $-105 \mathrm{~dB}$ and a shadowing standard deviation of 3.8 is used. Simulations are repeated 10 times and results are averaged. $\tau_{h}^{c}$ is set to $0.005 \mathrm{sec}$ for each channel $c$.

We use the case with non opportunistic spectrum access as the baseline in Fig. 2. For a scenario with $\tau_{s}^{c}$ spectrum sensing duration, and spectrum handoff instants $t_{c}$ for each channel $c$ as given in Table II, the number of received packets is examined. Three different estimation intervals $\left(\tau_{d}\right)$ are used to investigate the oscillations on the amount of received packets. Since adaptation to spectrum handoff is done at the end of decision interval, as this interval increases, adaptation gets delayed. This results in a decrease in the received packets. 


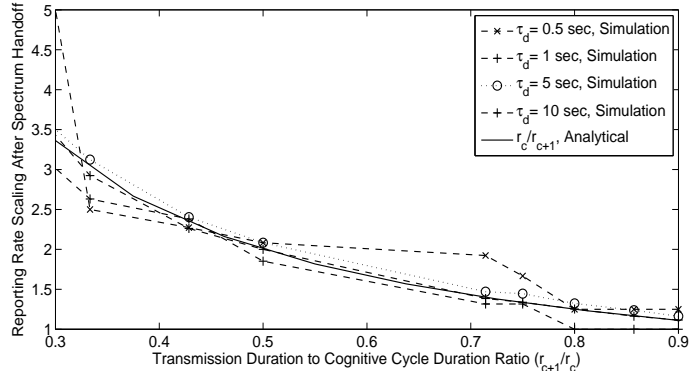

Fig. 4. Sensor node report rate update after spectrum handoff at next decision interval by sink for various $\tau_{d}$ values.

The effects of OSA on reliability, $\Gamma$, are presented in Fig. 3 for another scenario, with details as given in Table III. We define reliability as the ratio of received samples $X$ given in Eqn. (1), to desired number of samples, $X_{d}$ to satisfy minimum distortion $D_{0}$, i.e., $\Gamma=X / X_{d}$. Under OSA, there are significant oscillations in event observation reliability. This is caused by changes to $\tau_{t}^{c}$ and $\tau_{s}^{c}$ dictated by spectrum handoff. In Fig. 3, reliability oscillates about 20\%, and $40 \%$ for $\tau_{d}=0.5$ and $1 \mathrm{sec}$, respectively. Longer $\tau_{d}$ defers adapting the reporting rate due to spectrum handoff. Shorter decision interval cause more frequent rate updates and results in less performance loss after spectrum handoffs. As the decision intervals get longer, sink becomes less responsive to changes in spectrum parameters.

The ratio of data transmission to cognitive cycle duration in channel $c$, i.e., $\tau_{t}^{c} /\left(\tau_{t}^{c}+\tau_{s}^{c}\right)$, is defined as $r_{c}$. In Fig. 4, the reporting rate update performed by sink after spectrum handoff is presented for varying $r_{c+1} / r_{c}$ ratios, for different $\tau_{d}$. If a spectrum handoff is performed among channels with similar conditions, i.e., $r_{c+1} / r_{c}$ is about unity, then the rate update at the end of the estimation interval is about unity as well. When the ratio is smaller than 1 , reporting rate should be increased to meet the distortion criteria. Using the obtained rate regulation behavior in Fig. 4 for different $\tau_{d}$, reporting rate scaling after spectrum handoff is found as $\frac{1}{\Gamma_{k}} \approx \frac{r_{c+1}}{r_{c}}$.

We also depict alternative reporting rate update mechanisms in Fig. 4. For aggressive method, sensor rate updates are approximately $1 / r_{c}$. With this rate update, the transmission opportunity loss in the new channel is covered. The second one is the exponentially weighted moving average (EMWA) method, in which rate updates are calculated as

$$
R_{\mathrm{i}+1}=\alpha \frac{r_{c}}{r_{c+1}} R_{\mathrm{i}}+(1-\alpha) R_{\mathrm{i}}
$$

where $R_{\mathrm{i}+1}$ is the rate after update, $R_{\mathrm{i}}$ is the current rate, $\alpha$ is the forgetting factor, taken as 0.25 . It determines the impact of previous rates in determining the next rate. These two mechanisms will be discussed further in Section V-B.

These results motivate us to devise a spectrum-aware transport layer protocol that maintains the desired reliability in heterogeneous spectrum conditions. To achieve reliable distributed sensing, reporting rate updates should be decoupled from event estimation intervals. Reporting rates are updated after the spectrum handoff via spectrum-aware rate update, which provides a sufficient approximation for rate updates

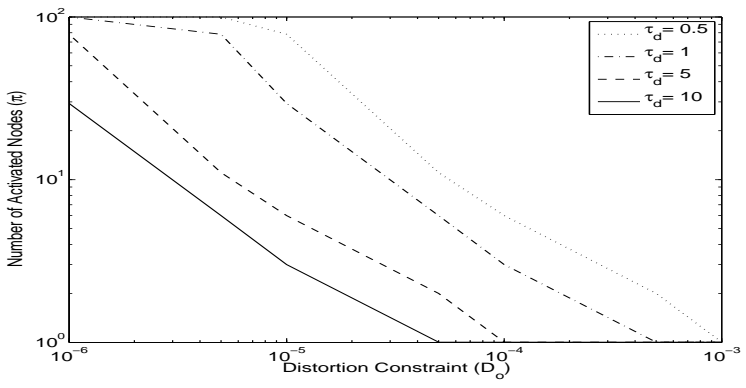

Fig. 5. Number of activated nodes with respect to distortion constraint $\left(D_{o}\right)$.

for different estimation intervals. We use this approach for regulation of the reporting rate in SERT and evaluate its performance in Section VI.

\section{B. Impact of Reliability Requirement on the Number of Re- porting Nodes}

We examine collaborative nature of CRSN for different distortion constraints and $\tau_{d}$ in Fig. 5. Dispersion loss $\gamma_{k}$ are selected randomly between 0.1 and 1 , and sensing noise $\xi_{m}$ is taken to be 0.01 for all sensing nodes. Simulations are run 1000 times and results are averaged. Minimum number of nodes required to satisfy $D_{0}$ for a given rate and $\tau_{d}$ using cost function in Eqn. (2) is plotted in log-log fashion.

The number of activated sensors increases with decreasing decision interval $\tau_{d}$. Cost function provided in Eqn. (2) enables sink to select sensors with higher SNR, minimizing event estimation distortion. Report rate can also be used to symbolize energy consumption limit per sensor. For the same energy consumption constraint, lower decision intervals require activating more source sensors. Therefore, selection of minimum distortion, i.e., maximum SNR sensors, is essential, when energy limitations and and variances in spectrum opportunities are considered. We consider both energy constraint and observation distortion of sensors in design of SERT. Along with rate control and congestion avoidance properties, SERT provides an SNR based source sensor activation algorithm to minimize energy consumption per decision interval of sensor nodes while conforming to the distortion requirement at sink.

TABLE II

LENGTHS OF $\tau_{s}^{c}$ AND $\tau_{t}^{c}$ FOR RECEIVED PACKet ANALysis

\begin{tabular}{c|c|c|c|c|c|c|c|c|c}
$\boldsymbol{c}$ & 1 & 2 & 3 & 4 & 5 & 6 & 7 & 8 & 9 \\
\hline$\tau_{\mathrm{t}}^{\mathrm{c}}(\mathrm{sec})$ & 0.3 & 0.6 & 0.3 & 0.2 & 0.6 & 0.3 & 0.4 & 0.2 & 0.6 \\
\hline$\tau_{\mathrm{s}}^{\mathrm{c}}(\mathrm{sec})$ & 0.2 & 0.05 & 0.2 & 0.05 & 0.05 & 0.2 & 0.1 & 0.1 & 0.1
\end{tabular}

TABLE III

LENGTHS OF $\tau_{s}^{c}$ AND $\tau_{t}^{c}$, AND SPECTRUM HANDOFF InSTANTS $\left(t_{c}\right)$ USED IN RELIABILITY ANALYSIS

\begin{tabular}{c|c|c|c|c|c|c|c|c|c|c}
$\boldsymbol{c}$ & 1 & 2 & 3 & 4 & 5 & 6 & 7 & 8 & 9 & 10 \\
\hline$\tau_{\mathbf{t}}^{\mathbf{c}}$ & 0.6 & 0.3 & 0.6 & 0.3 & 0.2 & 0.6 & 0.3 & 0.4 & 0.2 & 0.6 \\
\hline$\tau_{\mathbf{s}}^{\mathbf{c}}$ & 0.1 & 0.2 & 0.05 & 0.2 & 0.05 & 0.05 & 0.2 & 0.1 & 0.1 & 0.1 \\
\hline $\mathbf{t}_{\mathbf{c}}$ & 0 & 3 & 5 & 11 & 14 & 15 & 17 & 22 & 25 & 28
\end{tabular}




\section{Impact of Spectrum Coordination on Report Rate in CRSN}

In [15], simulations on the performance of event delivery in case of spectrum coordination failures are presented. Certain percentage of the source nodes in the event region are left uncoordinated after spectrum handoff to assess reliability performance of existing works. While event reliability oriented protocols [13], [14] update their reporting rate to satisfy reliability constraints, congestion control oriented protocols [9], [17] do not keep up with reliability requirements and every sensor regulates its reporting rate irrespective to event detection and tracking requirements. However, in reliability oriented approaches, reporting nodes' energy consumption is increased to satisfy reliability constraint since reporting rates of coordinated sensor nodes are updated. This shows that network lifetime is highly dependent on spectrum coordination.

Moreover, PU communication disrupts certain number of sensors participating in event delivery, where desired number of samples should be obtained by the remaining sensors. Thus, the the burden on the sensors is based on spectrum opportunity. While collecting required number of samples from sensors to meet the desired distortion metric $D_{0}$, it is essential to limit the reporting rate of sensors and benefit from estimation diversity. Thus, we propose a source sensor opportunity-balancing algorithm in terms of reporting rate, based on observation noise of sensors. With each disruption in reporting sensors, a new desired sample amount according to observation noise of remaining sensors are selected jointly.

\section{SERT: SPECTRUM-AWARE ENERGY-ADAPTIVE RELIABlE TRANSPORT PROTOCOL FOR CRSN}

We aim to adapt to PU activity rapidly while providing a sensor data report rate to meet $D_{0}$. To this end, SERT has a number of functionalities as detailed below.

\section{A. Instant Start}

Upon event detection, sensors must instantly respond to the phenomenon in terms of event delivery. Therefore, sensors are required to start event delivery at a rate that sink can react properly in a desired interval. On the other hand, dense deployment and bursty event arrivals can cause severe congestion. Dynamically changing spectrum environment is also critical in starting delivery of an instantly happening event.

Based on observed event statistics, total sample collecting rate per decision interval to reliably observe event features at sink is equal to $S_{d}$. Sensor nodes send their SNR $\left(\psi_{m}\right)$ to sink and SNR threshold for reporting sensor nodes, $\psi_{t}$, is set accordingly. Then, sensor nodes that have SNR greater than $\psi_{t}$ start event reporting. Details related to threshold determination are given in Section V-C. Initial report rate for source nodes $R_{i}$ is evaluated as $R_{\text {initial }}=\frac{S_{d}}{r_{c} \cdot \tau_{d} \cdot M \cdot S_{p}}$.

\section{B. Spectrum-aware Report Rate Control}

Reliability is controlled at each $\tau_{d}$. In case of lower reliability without congestion, reporting rate is scaled to achieve desired total received number of samples at sink. This notion of spectrum-aware reliability distinguishes SERT from other
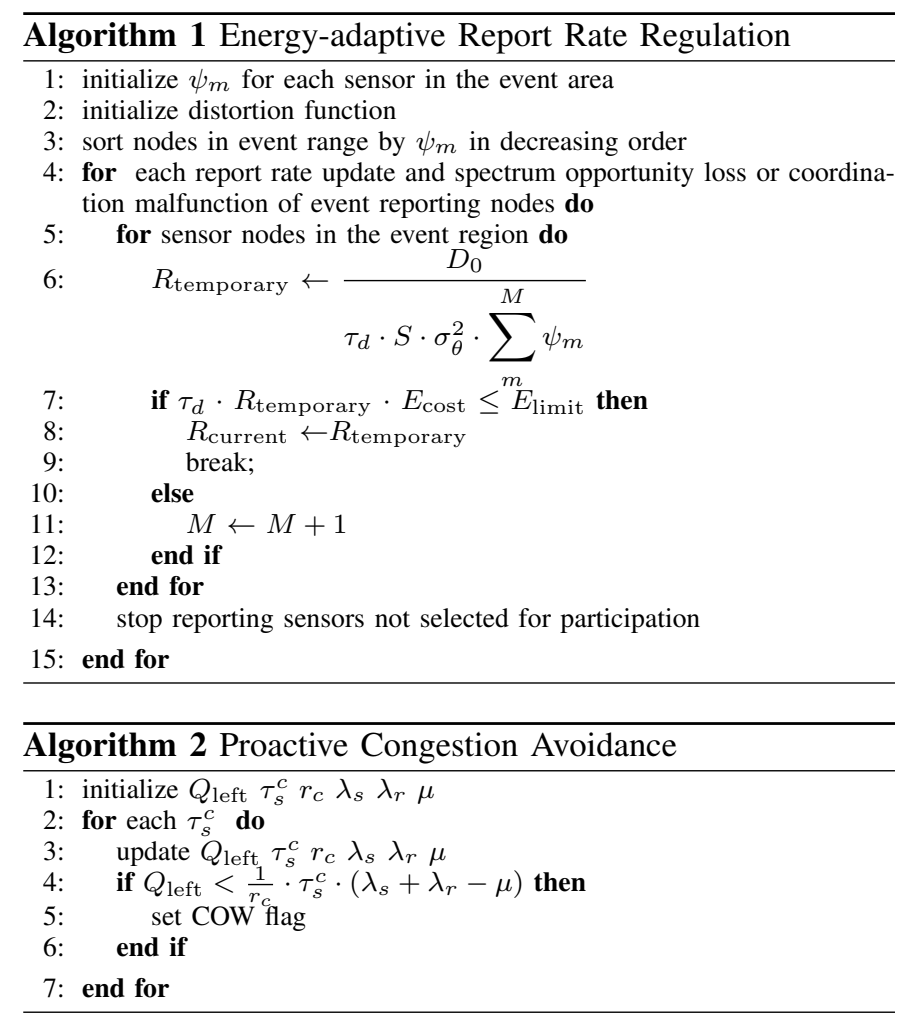

existing transport layer models that focus on fixed spectrum access. As shown in Fig. 4, at each $\tau_{d}$, the report rate of sensors are updated to compensate for loss of transmission opportunity due to spectrum sensing. However, when multiple spectrum handoffs take place, update at event decision becomes inaccurate as shown in Fig. 3. Moreover, for larger $\tau_{d}$, $D_{0}$ cannot be satisfied while waiting for a rate update and this causes unreliable distributed sensing operation.

In Fig. 4, two different curves for rate update as well as simulation results are presented, namely, aggressive approach and Exponentially Weighted Moving Average (EWMA) approach. In aggressive approach, rates updates are matched approximately with $1 / r_{c}$ to recover transmission opportunity loss due to spectrum handoff. In Fig. 4, we show, for various $\tau_{d}$, via simulation results that $1 / r_{c}$ scaling almost consistent with decision interval scaling after spectrum handoff. Alternatively, a soft update approach, EWMA is also presented. Compared to the aggressive approach, it provides a reduced recovery with consideration of frequent spectrum handoffs and congestion.

\section{Reliable Life Time via Collaborative Energy Adaptation}

SERT achieves reliable network lifetime via adjusting node report rates to satisfy reliability requirement with minimum number of nodes. Moreover, relaying nodes reject relaying new flows if their energy consumption limit per $\tau_{d}$ is reached.

$$
\begin{array}{ll}
\min & M \\
\text { s.t. } & \left(\sum_{n=1}^{M} \frac{\gamma_{n}^{2}}{\xi_{n}^{2}}\right)^{-1}<D_{0} \\
& \tau_{d} \cdot R_{\text {current }} \cdot E_{\text {cost }}<E_{\text {limit }}
\end{array}
$$




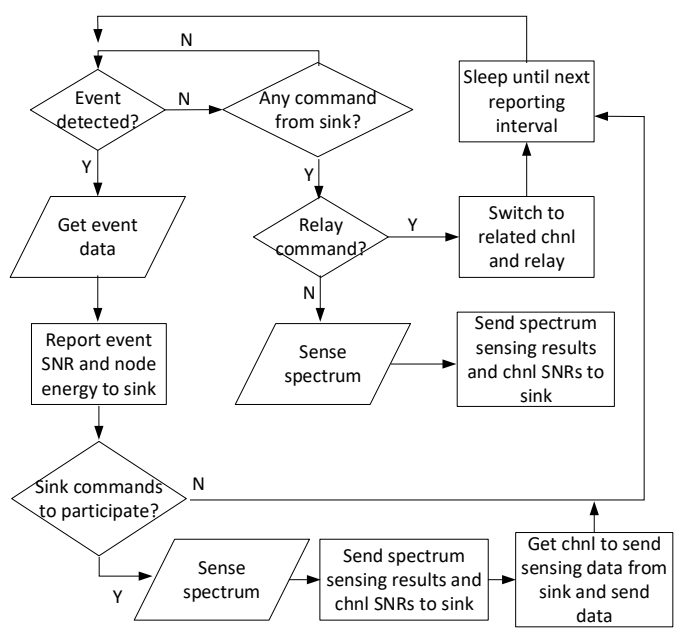

(a)

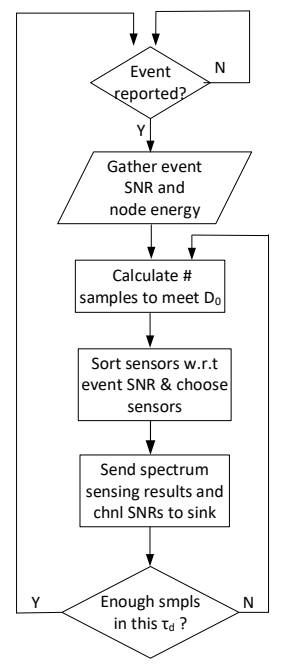

(b)

Fig. 6. Flowchart of overall SERT operation for (a) sensor nodes (b) the sink node

Sensors are ordered according to their descending event SNR values. A threshold SNR, $\psi_{t}$, is determined which will enable sufficient amount of source nodes to send event data so that the reliability constraint can be met. This threshold is set by the energy-adaptive in Algorithm 1.

\section{Proactive Congestion Avoidance}

Proactive congestion detection is achieved by piggybacking congestion information in the header of packets. By listening to the channel, child nodes can capture such information when packets including congestion on the way flag (COW) are forwarded by their parent nodes towards the sink.

Congestion is detected if $Q_{\text {left }}<\frac{1}{r_{c}} \cdot \tau_{s}^{c} \cdot\left(\lambda_{s}+\lambda_{r}-\mu\right)$ where $Q_{\text {left }}$ is the size of empty slots in queue, $\tau_{s}^{c}$ is the spectrum sensing duration for channel $c$ and it is selected as queue decision duration for proactive congestion avoidance, $\lambda_{s}$ is the queue incoming rate due to sensing in source nodes, and $\lambda_{r}$ is the queue incoming rate due to relaying. Congestion avoidance algorithm is detailed in Algorithm 2.

This mechanism is incorporated into SERT through hopby-hop congestion control for nodes that participate in forwarding packets as long as the requirement for $Q_{\text {left }}$ is not satisfied. The implementation of the inequality necessitates calculation of the parameters $\tau_{s}^{c}, \lambda_{s}, \lambda_{r}$, and $\mu$ at sensor nodes participating in event delivery. $\lambda_{s}$ is easily extracted from the rate of injected packets from the sensing boards to the communication module. $\tau_{s}^{c}$ is obtained from cross-layer functionality and remains constant until spectrum handoff. Consequently, each node updates these values as event-tosink transport is carried on. When congestion is detected, COW flag is set in the forwarded packets and piggybacked to child nodes and parent nodes. When alarmed for congestion avoidance, COW receiving nodes check for alternative route. If an alternative route is not available, relaying is stopped, and if it is a source node, it stops packet generation as well. Overall, SERT operation flowchart is given in Fig. 6.

\section{SERT PERFormanCE EVALUATION}

In this section, performance evaluations of SERT for spectrum mobility and reliable life time are performed. In spectrum mobility section, we present comparison of SERT with the closest possible transport protocol in the literature. In reliable life time section, we show the performance gain achieved by proposed node selection scheme.

We calculate energy consumption as $E_{l}=\tau_{d} \cdot I_{l} \cdot V$ and $E_{t}=T_{b} \cdot L_{p} \cdot I_{t} \cdot V$, where $E_{l}$, and $E_{t}$ are energy spent for listening mode, and for transmission of a packet, respectively. $I_{l}$ is the listening mode current, $I_{t}$ is the transmitting current, $T_{b}$ is the byte transmission time, $L_{p}$ is the packet size, and $V$ is the supply voltage of the sensor nodes [29]. The values used in the simulation are $3 \mathrm{~V}$ supply voltage, $I_{l}=18.8 \mathrm{~mA}$, $I_{t}=17.4 \mathrm{~mA}, T_{b}=32 \mu \mathrm{sec}, L_{p}=64$ Bytes.

\section{A. Spectrum Mobility}

To the best of our knowledge, there is no existing transport protocol that addresses challenges due to incorporation of CR functionality into sensor nodes. Moreover, protocols devised for conventional sensor networks cannot be compared with SERT in a fair manner, since they do not have spectrum awareness. We use (RT) ${ }^{2}$ [14] for comparison due to its reliabilityoriented rate control and investigate how it compares to SERT in adapting to maintain reliability after spectrum handoff. For cases where $r_{c+1}$ is greater than $r_{c}$, rate adaptation is not necessary, since transmission time is greater in the new channel

TABLE IV

CHANNEL ACCESS INTERVALS IN TERMS OF $\tau_{d}$

\begin{tabular}{c|c|c|c|c|c|}
\multicolumn{2}{c}{} & \multicolumn{5}{c}{ Channel $(\boldsymbol{c})$} \\
\cline { 3 - 6 } & 1 & 2 & 3 & 4 \\
\cline { 2 - 6 } & $\tau_{d}=0.5 \mathrm{sec}$ & $1-3$ & $3-4$ & $4-6$ & $6-8$ \\
\cline { 2 - 6 } $\begin{array}{c}\text { Decision } \\
\text { Interval }\end{array}$ & $\tau_{d}=1 \mathrm{sec}$ & $1-3$ & $3-5$ & - & $5-7$ \\
\cline { 2 - 6 } & $\tau_{d}=5 \mathrm{sec}$ & $1-2$ & $2-3$ & $3-4$ & $4-5$ \\
\cline { 2 - 6 } & $\tau_{d}=10 \mathrm{sec}$ & $1-1.5$ & $1.5-2$ & $2-2.5$ & $2.5-3$ \\
\cline { 2 - 6 } & & &
\end{tabular}




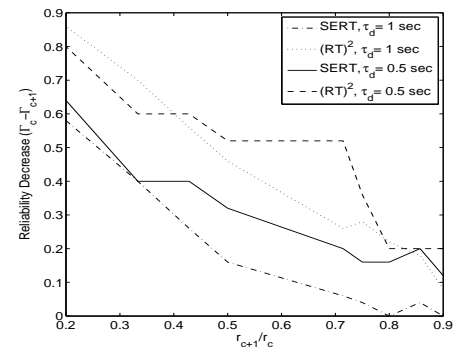

(a)

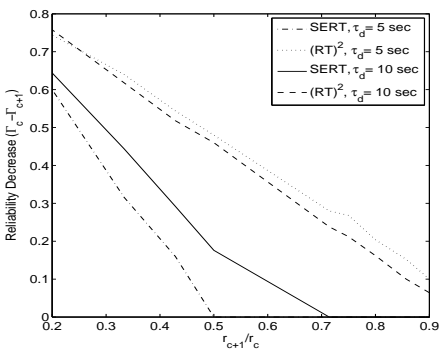

(b)

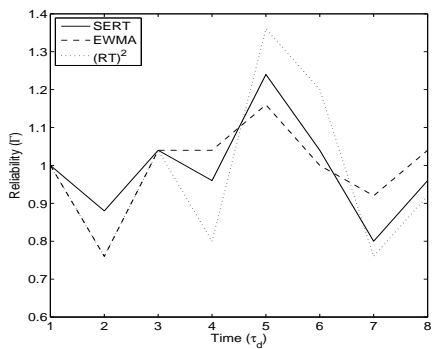

(c)

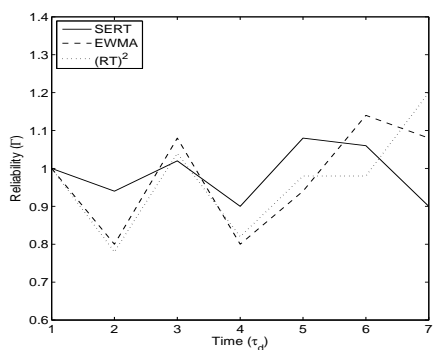

(d)

Fig. 7. Comparison of reliability performance between SERT and (RT) ${ }^{2}$ after spectrum handoff for various $\tau_{d}$ values. (a) For $\tau_{d}=0.5$ sec. and $\tau_{d}=1$ sec. (b) For $\tau_{d}=5 \mathrm{sec}$. and $\tau_{d}=10 \mathrm{sec}$ and Transient reliability performance of SERT for $\tau_{d}$ values of 0.5 and $1 \mathrm{sec}$, in (c) and (d), respectively.

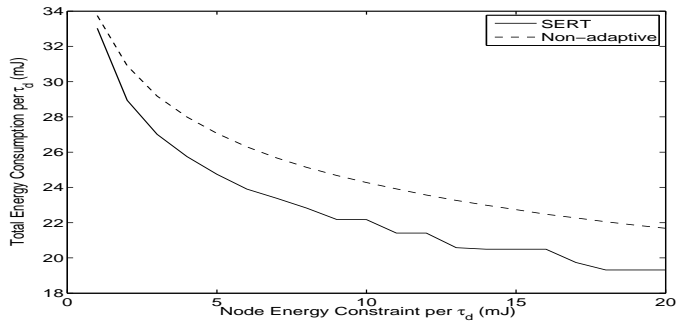

(a)

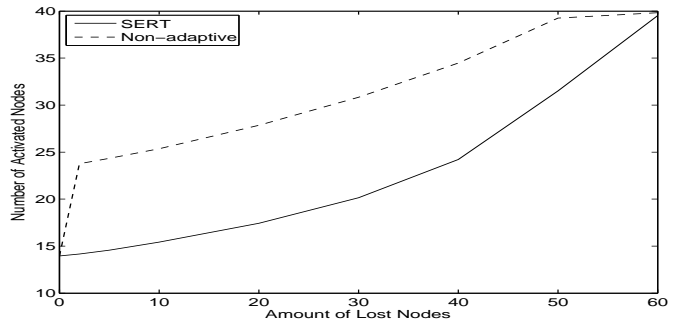

(b)

Fig. 8. (a) Total energy consumption with respect to available per node energy. (b) Number of activated nodes with respect to number of lost nodes.

and consequently, sending sufficient amount of sensing data to meet the distortion requirement is always possible. Therefore, we investigate the effect of spectrum handoff when $r_{c+1}$ is smaller than $r_{c}$, i.e. there are less transmission time in the new channel. $r_{c+1} / r_{c}$ value is varied from 0.2 to 0.9 .

We use 4 channels and 4 different values of $\tau_{d}=0.5,1,5$ and 10 secs. For transient reliability analysis, $\tau_{t}^{c}$ durations are set to $0.4,0.2,0.5,0.3 \mathrm{sec}$ for channels 1 to 4 , respectively, while $\tau_{s}^{c}$ durations are set to 0.1 for all channels. PU arrivals are scheduled randomly. Reliability requirements for $\tau_{d}=0.5$, 1,5 and 10 secs are set as 25,50,250, 500, packets per $\tau_{d}$, respectively. Channel access intervals are given in Table IV.

1) Reliability Decrease After Spectrum Handoff: In Fig. 7, we show how reliability changes as the ratio of the transmission duration to cognitive cycle duration $\left(r_{c+1} / r_{c}\right)$ changes. Generally speaking, as $r_{c+1} / r_{c}$ decreases, we have higher decrease in reliability. We see that SERT provides the least amount of change in reliability after a spectrum handoff, since SERT updates its rate at spectrum handoff, while (RT) ${ }^{2}$ waits for the end of estimation interval and attributes the reliability decrease to node failures and packet losses. Furthermore, SERT experiences no reliability loss for $r_{c+1} / r_{c}$ values greater than 0.5 for $\tau_{d}=5$ and $0.7 \mathrm{sec}$ for $\tau_{d}=10 \mathrm{sec}$. The variation of reliability after spectrum handoff in Fig. 7 shows that SERT responds to the varying $r_{c}$ with less variation in reliability.

2) Transient Reliability Performance: As an alternative conservative rate update policy to compare with reliability performance of SERT, we use exponentially weighted moving average (EWMA) in addition to (RT) ${ }^{2}$, where we take, $\alpha=0.25$ for EWMA rate update. SERT rate update can also be derived from EWMA via setting $\alpha=1$. In Fig. 7(a) and (b), the transient reliability performance of SERT is presented, where consecutive spectrum handoffs cause a change of cognitive cycle parameters, changing the received number of samples from sensors at the sink. This change dominates the reliability performance and prevents fixing the reporting rate of sensors. However, SERT achieves reduced reliability variations around \pm 0.2 and less than \pm 0.1 for $\tau_{d}=0.5$ and $1 \mathrm{sec}$, respectively. For $\tau_{d}=1 \mathrm{sec}$ in Fig. 7(a), SERT achieves a significantly higher reliability stability due to the spectrum-aware rate control mechanism. Although EWMA rate update scheme performs close to SERT for $\tau_{d}=0.5 \mathrm{sec}$, its conservative policy does not provide sufficient adaptation and for $\tau_{d}=1 \mathrm{sec}$, it performs far below SERT and close to $(\mathrm{RT})^{2}$. (RT) $)^{2}$ updates reporting rate at the end of each estimation period in accordance with its rate regulation policy. It fails to adapt changing spectrum conditions, and as the estimation interval gets higher compared to $\tau_{t}^{c}+\tau_{s}^{c}$, its reliability performance falls behind SERT dramatically.

The effect of the spectrum-aware rate control results in higher transient reliability performance for SERT as seen in Fig. 7(c) \& (d). Furthermore, the performance improves when the difference between the $r_{c}$ value of the channels is higher, implying that spectrum-aware rate control is effective in achieving reliability. The transient variation of the reliability shows that SERT responds to spectrum handoff functionality immediately, and after the rate update the reliability is achieved in most of the cases, while oscillations are reduced.

\section{B. Reliable Life Time}

In Fig. 8(a), relationship between node energy constraint and total energy consumption per $\tau_{d}$ is given. $D_{0}$ is set to $10^{-4}$. It is seen that while the energy level per node increases, total energy consumption in network decreases, since the 
number of activated nodes is decreased. Moreover, SERT provides about $5 \mathrm{dBm}$ energy gain per decision interval for $20 \mathrm{~mJ}$ energy level per $\tau_{d}$ with its SNR aware sensor node selection and rate determination algorithm. This gain provides extended reliable life time for sensor network.

In Fig. 8(b), number of activated nodes $(\zeta)$ is revealed compared to random replacement source node selection. As the percentage of lost sensor nodes in the event region increases, the number of activated sensor nodes also increases, to compensate for lost nodes. SERT provides SNR based source activation, and hence, it provides the same amount of distortion level for less amount of activated source sensors compared to random source node selection case. The difference of number of activated nodes varies around from 5 to 10 .

\section{CONCLUSION}

SERT provides spectrum-aware reliable event transport with energy-adaptive source activation and rate limitation. With introduced enhancements, source nodes control their reporting rate via decision intervals, which are adapted to OSA and varying spectrum characteristics as well as energy constraints to satisfy reliability at sink. Simulation results show that SERT adapts OSA environment, and provides reliable event delivery in CRSN while preserving unnecessary consumption of limited energy of sensor nodes.

\section{REFERENCES}

[1] S. Haykin, "Cognitive Radio: Brain-empowered Wireless Communications", IEEE Journal on Selected Areas in Communications (JSAC), Vol. 23, No. 2, pp. 201-220, Feb. 2005.

[2] O. B. Akan, O. B. Karli, and O. Ergul, "Cognitive Radio Sensor Networks," IEEE Network, vol. 23, no. 4, pp. 34-40, July-August 2009

[3] M. Sujeethnanda, R. M. Garimella, "Mobile Cognitive Wireless Sensor Networks: Precision Agriculture," Proc. Agro-Informatics and Precision Agriculture, 2012.

[4] O. Ergul, G. A. Shah, B. Canberk, O. B. Akan, "Adaptive and Cognitive Communication Architecture for Next-generation PPDR Systems," IEEE Communications Magazine, vol. 54, no. 4, pp. 92-100, April 2016.

[5] O. Ergul, A. O. Bicen, O. B. Akan, "Opportunistic Reliability for Sensor Actor Networks in Smart Grid," Ad hoc Networks Journal (Elsevier), vol. 41, pp. 5-14, May 2016.

[6] J. A. Han, W. S. Jeon, and D. G. Jeong, "Energy-Efficient Channel Management Scheme for Cognitive Radio Sensor Networks," IEEE Trans. on Vehicular Tech., vol. 60, no. 4, pp. 1905-1910, May 2011.

[7] O. Ergul, O. B. Akan, "Cooperative Coarse Spectrum Sensing for Cognitive Radio Sensor Networks," in Proc. IEEE WCNC, pp. 20552060, April 2014.

[8] O. Ergul, A. O. Bicen, O. B. Akan, "Energy-efficient Cooperative Spectrum Sensing for Cognitive Radio Sensor Networks," in Proc. IEEE ISCC, pp. 465-4694, Juky 2013.

[9] C. Wan, S. B. Eisenman, and A.T. Campbell, "CODA: Congestion Detection and Avoidance in Sensor Networks," in Proc. ACM Sensys, Los Angeles, USA, Nov. 2003.

[10] M. Zawodniok, and S. Jagannathan, "Predictive Congestion Control Protocol for Wireless Sensor Networks," IEEE Trans. on Wireless Communications, vol. 58, no. 9, pp. 3955-3963, Nov. 2007.

[11] C. Wang, B. Li, K. Sohraby, M. Daneshmand, and Y. Hu, "Upstream Congestion Control in Wireless Sensor Networks Through Cross-Layer Optimization," IEEE Journl of Selected Areas in Communications, vol. 25, no. 4, pp. 786-795, May 2007.

[12] F. Stan and J. Heidemann, "RMST: Reliable Data Transport in Sensor Networks," in Proc. IEEE SNPA 2003, pp. 102-112, May 2003.

[13] O. B. Akan and I. F. Akyildiz, "Event-to-Sink Reliable Transport in Wireless Sensor Networks," IEEE/ACM Trans. Netw., vol. 13, no. 5, pp. 1003-1016, Oct. 2005.
[14] V. C. Gungor, O. B. Akan, and I. F. Akyildiz, "A Real-Time and Reliable Transport Protocol for Wireless Sensor and Actor Networks," IEEE/ACM Trans. on Netw., vol. 16, no. 2, pp. 359-370, Apr. 2008.

[15] A. O. Bicen, and O. B. Akan, "Reliability and Congestion Control in Cognitive Radio Sensor Networks," Elsevier Ad Hoc Netw. J., vol. 9, no. 7, pp. 1154-1164, Sept. 2011.

[16] D. Sarkar, and H. Narayan, "Transport Layer Protocols for Cognitive Networks," in Proc. IEEE INFOCOM 2010, USA, Mar. 2010.

[17] K. R. Chowdhury, M. Di Felice, and I. F. Akyildiz, "TCP CRAHN: A Transport Protocol for Cognitive Radio Ad-Hoc Networks," IEEE Trans. on Mobile Computing, vol. 12, no. 4, pp. 790-803, Apr. 2013.

[18] C. Luo, F. R. Yu, H. Ji, and V. C. M. Leung, "Cross-Layer Design for TCP Performance Improvement in Cognitive Radio Networks," IEEE Trans. on Vehicular Technology, vol. 59, no. 5, pp. 2485-2495, Jun. 2010.

[19] D. Chen, H. Ji, and V. C. M. Leung, "Distributed Best-Relay Selection for Improving TCP Performance Over Cognitive Radio Networks: A Cross-Layer Design Approach," IEEE Journal of Selected Areas in Communications, vol. 30, no. 2, pp. 315-322, Feb. 2012.

[20] A. O. Bicen, and O. B. Akan, "TFRC-CR: An Equation-based Transport Protocol for Cognitive Radio Networks," Elsevier Ad Hoc Netw. J., vol. 11, no. 6, pp. 1836-1847, Aug. 2013.

[21] K. Tsukamoto, S. Koba, M. Tsuru, Y. Oie, "Cognitive Radio-Aware Transport Protocol for Mobile Ad Hoc Networks," IEEE Trans. Mob. Comput. vol. 14, no. 2, pp. 228-301, Feb. 2015.

[22] X. X. Zhong, Y. Qin, L. Li, "Transport Protocols in Cognitive Radio Networks: A Survey," KSII Trans. Internet and Information Systems vol. 8, pp. 3711-3730, Nov. 2014.

[23] S. Salim, S. Moh, "A Robust and Energy-Efficient Transport Protocol for Cognitive Radio Sensor Networks," Sensors J., vol. 14, no. 10, pp. 19533-19550, Oct. 2014.

[24] Y. B. Zikria, S. Nosheen, F. Ishmanov, S. W. Kim, "Opportunistic Hybrid Transport Protocol (OHTP) for Cognitive Radio Ad Hoc Sensor Networks," Sensors J., vol. 15, no. 12, pp. 31672-31686, Dec. 2015.

[25] M. D. Felicea, K. R. Chowdhury, W. Kimc, A. Kassler, L. Bononi, "Endto-end Protocols for Cognitive Radio Ad Hoc Networks: An Evaluation Study," Elsevier Perf. Eval. vol. 68, no. 12, pp. 859-875, Dec. 2011.

[26] J. M. Mendel, Lessons in Estimation Theory for Signal Processing, Communications, and Control.Englewood Cliffs, NJ: Prentice-Hall, 1995.

[27] The Network Simulator, ns-3 [Online]. Available: http://www.nsnam.org

[28] M. Zuniga, B. Krishnamachari, "Analyzing the Transitional Region in Low Power Wireless Links," in Proc. IEEE SECON, pp. 517-526, 2004

[29] R. Jurdak, A. G. Ruzelli, and G. M. P. O'Hare, "Radio Sleep Mode Optimization in Wireless Sensor Networks," IEEE Transactions on Mobile Computing, vol.9, no.7, pp. 995-968, Jul. 2010. 